\title{
Factors Influencing Progress toward Ecological Speciation
}

\author{
Marianne Elias, ${ }^{1}$ Rui Faria, ${ }^{2,3}$ Zachariah Gompert, ${ }^{4}$ and Andrew Hendry ${ }^{5}$ \\ ${ }^{1}$ CNRS, UMR 7205, Muséum National d'Histoire Naturelle, 45 Rue Buffon, CP50, 75005 Paris, France \\ ${ }^{2}$ CIBIO/UP — Centro de Investigação em Biodiversidade e Recursos Genéticos, Universidade do Porto, Campus Agrário de Vairão, \\ R. Monte-Crasto, 4485-661 Vairão, Portugal \\ ${ }^{3}$ IBE_-Institut de Biologia Evolutiva (UPF-CSIC), Universitat Pompeu Fabra, PRBB, Avenue Doctor Aiguader N88, \\ 08003 Barcelona, Spain \\ ${ }^{4}$ Department of Botany, 3165, University of Wyoming, 1000 East University Avenue Laramie, WY 82071, USA \\ ${ }^{5}$ Redpath Museum and Department of Biology, McGill University, 859 Sherbrooke Street West Montreal, QC, Canada H3A 2 K6
}

Correspondence should be addressed to Marianne Elias, melias2008@googlemail.com

Received 28 March 2012; Accepted 28 March 2012

Copyright (C) 2012 Marianne Elias et al. This is an open access article distributed under the Creative Commons Attribution License, which permits unrestricted use, distribution, and reproduction in any medium, provided the original work is properly cited.

\section{Introduction}

Ecological speciation occurs when adaptation to divergent environments, such as different resources or habitats, leads to the evolution of reproductive isolation $[1,2]$. More specifically, divergent (or disruptive) selection between environments causes the adaptive divergence of populations, which leads to the evolution of reproductive barriers that decrease, and ultimately cease, gene flow [3, 4]. Supported by a growing number of specific examples, ecological speciation is thought to be a primary driving force in evolutionary diversification, exemplified most obviously in adaptive radiations [5-8].

As acceptance of the importance of ecological speciation has grown, so too has the recognition that it is not all powerful. Specifically, a number of instances of nonecological speciation and nonadaptive radiation seem likely [9], and colonization of different environments does not always lead to speciation $[10,11]$. This latter point is obvious when one recognizes that although essentially all species are composed of a number of populations occupying divergent environments [12], only a fraction of these ever spin off to become full-fledged species. Instead, populations occupying divergent environments or using different resources show varying levels of progress toward ecological speciation-and this variation provides the substrate to study factors that promote and constrain progress along the speciation continuum. By studying these factors, we can begin to understand why there are so many species [13] and also why there are so few species [14].

This special issue on ecological speciation puts snapshots of progress toward speciation sharply in focus and then investigates this topic from several angles. First, several papers provide conceptual or theoretical models for how to consider progress toward ecological speciation (Funk; Heard; Lenormand; Liancourt et al.; Agrawal et al.). Second, several papers highlight the noninevitability of ecological speciation through investigations where ecological speciation seems to be strongly constrained (Räsänen et al.; Bolnick) or at least lacking definitive evidence (Ostevik et al.; Scholl et al.). Some of these papers also uncover specific factors that seem particularly important to ecological speciation, such as the combination of geographic isolation and habitat differences (Surget-Groba et al.), the strength of disruptive selection and assortative mating (Bolnick), and host-plant adaptation (Scholl et al.). Third, several particularly important factors emerge as a common theme across multiple papers, particularly parasites/pollinators (Xu et al.; Karvonen and Seehausen), habitat choice (Webster et al.; Feder et al.; Carling and Thomassen; Egan et al.), and phenotypic plasticity (Fitzpatrick; Vallin and Qvarnström).

Here we highlight the most important aspects of these contributions and how they relate to three major topics: (i) models for progress toward ecological speciation; (ii) variable progress toward ecological speciation in nature; and (iii) factors affecting progress toward ecological speciation. 


\section{Models for Progress toward Ecological Speciation}

Terminological issues have long bedevilled communication among researchers working on speciation. D. J. Funk addresses this topic by first clarifying the relationship between sympatric speciation (whereby reproductively isolated populations evolve from an initially panmictic population) and ecological speciation (whereby reproductive isolation evolves as a consequence of divergent/disruptive natural selection). These are orthogonal concepts [15]. First, even if disruptive selection is a common way of achieving sympatric speciation, this can also be caused by other factors, such as changes in chromosome number. Second, ecological speciation can readily occur in allopatry $[16,17]$. Funk then introduces four new concepts aiming to reduce confusion in the literature. Sympatric race is a generalisation of host race (usually used for herbivores or parasites) and refers to any sympatric populations that experience divergent selection and are partly but incompletely reproductively isolated. Envirotypes are populations that differ due to phenotypic plasticity. Host forms are populations that exhibit host-associated variation, but for which the nature of variation (e.g., envirotype, host race, cryptic species) has not yet been diagnosed. Ecological forms are a generalization of host forms for nonherbivore or parasitic taxa. The two latter concepts acknowledge the fact that one has an incomplete understanding of speciation. To overcome the problem of overdiagnosing host races, Funk introduces five criteria, based on host association and choice, coexistence pattern, genetic differentiation, mate choice, gene flow, and hybrid unfitness. Funk's maple and willow associated phytophagous populations of Neochlamisus bebbianae leaf beetle meet all these criteria and can, therefore, be considered as host races.

Another phytophagy-inspired conceptual model for how an insect species initially using one plant species might diversify into multiple insect species using different host plants is presented by $S$. Heard. This effort explicitly links variation in host plant use within insect species or races to the formation of different host races and species. In this proposed "gapeand-pinch" model, Heard posits four stages (or "hypotheses") of diversification defined in part by overlap in the plant trait space used by the insect races/species. In the first stage "adjacent errors," some individuals within an insect species using one plant species might "mistakenly" use individuals of another plant species that have similar trait values to their normal host plant species. In the next stage "adjacent oligophagy," populations formed by the insects that shifted plant species then experience divergent selection-and undergo adaptive divergence- - leading to a better use of that new host. In the third stage "trait distance-divergence," competition and reproductive interactions cause character displacement between the emerging insect races or species so that they become specialized on particularly divergent subsets of the trait distributions of the two plant species. In the final stage "distance relaxation," the new species become so divergent that they no longer interact, and can then evolve to use trait values more typical of each plant species. Heard provides a theoretical and statistical framework for testing this model and applies it to insects using goldenrod plants.

Local adaptation is often the first step in ecological speciation, and so factors influencing local adaptation will be critical for ecological speciation. Local adaptation can either increase over time (if more specialized alleles spread), eventually leading to speciation, or it can decrease over time (if more generalist alleles spread). T. Lenormand reviews the conditions that favor these different scenarios and emphasizes the role of three positive feedback loops that favor increased specialization. In the demographic loop, local adaptation results in higher population density, which in turn favors the recruitment of new locally adapted alleles. In the recombination loop, locally adapted alleles are more likely to be recruited in genomic regions already harbouring loci with locally adapted alleles, thereby generating genomic regions of particular importance to local adaptation. In the reinforcement loop, local adaptation selects for traits that promote premating isolation (reinforcement), which in turn increases the recruitment and frequency of locally adapted alleles. Lenormand then details the mechanisms involved in reinforcement, particularly assortative mating, dispersal, and recombination. He highlights that these characteristics represent the three fundamental steps in a sexual life cycle (syngamy, dispersal, and meiosis) and that they promote genetic clustering at several levels (within locus, among individuals, among loci). His new classification is orthogonal to, and complements, the traditional one- versus two-allele distinction [14]. Overall, the rates of increased specialization and reinforcement determine progress toward ecological speciation.

One of the major constraints on ecological speciation is the establishment of self-sustaining populations in new/ marginal environments, because the colonizing individuals are presumably poorly adapted to the new conditions. This difficulty might be eased through facilitation, the amelioration of habitat conditions by the presence of neighbouring living organisms (biotic components) [18]. According to this process, the benefactor's "environmental bubble" facilitates the beneficiary's adaptation to marginal conditions, which can result in ecological speciation if gene flow from the core habitat is further reduced. At the same time, however, facilitation might hinder further progress toward ecological speciation by maintaining gene flow between environments and by preventing reinforcement in secondary contact zones. P. Liancourt, P. Choler, N. Gross, X. Thibert-Plante, and K. Tielbörg consider these possibilities from the beneficiary species perspective, through a spatially and genetically explicit modelling framework that builds on earlier models $[19,20]$. They find that ecological speciation is more likely with larger patch (facilitated versus harsh) sizes. Liancourt and coauthors further suggest that facilitation can play another important role in evolution by helping to maintain a genetic diversity "storage" in marginal habitats, a process with some parallel to niche conservationism. A deeper understanding of the role of facilitation in diversification is needed (both theoretically and empirically), and the authors suggest that stressful environmental gradients would be useful study systems for this endeavour. 
Intrinsic postzygotic isolation, a fundamental contributor to speciation, is often caused by between-locus genetic incompatibilities $[21,22]$. The origin of these incompatibilities, particularly in the face of gene flow, remains an outstanding question. A. F. Agrawal, J. L. Feder, and P. Nosil use two-locus two-population mathematical models to explore scenarios where loci subject to divergent selection also affect intrinsic isolation, either directly or via linkage disequilibrium with other loci. They quantified genetic differentiation (allelic frequencies of loci under selection), the extent of intrinsic isolation (hybrid fitness), and the overall barrier to gene flow (based on neutral loci). They find that divergent selection can overcome gene flow and favors the evolution of intrinsic isolation, as suggested previously [23]. Counterintuitively, intrinsic isolation can sometimes weaken the barrier to gene flow, depending on the degree of linkage between the two focal loci. This occurs because intrinsic isolation sometimes prevents differentiation by divergent selection.

\section{Variable Progress toward Ecological Speciation in Nature}

Threespine stickleback fish, with their diverse populations adapted to different habitats, had provided a number of examples of how adaptive divergence can promote ecological speciation $[24,25]$. Indeed, work on this group has fundamentally shaped our modern understanding of ecological speciation [1-3]. At the same time, however, three-spine stickleback also provides evidence of the frequent failure of divergent selection to drive substantial progress toward ecological speciation [25]. This special issue provides two of such examples. In one, K. Räsänen, M. Delcourt, L. J. Chapman and A. P. Hendry report that, despite strong divergent selection, lake and stream stickleback from the Misty watershed do not exhibit positive assortative mate choice in laboratory experiments. These results are in contrast to the strong assortative mating observed in similar studies of other stickleback systems, such as benthic versus limnetic [26] and marine versus fresh water [27]. In addition to providing potential explanations for this discrepancy, Räsänen et al. conclude that the apparent conundrum of limited gene flow but no obvious reproductive barriers could be very informative about the factors that constrain progress toward ecological speciation.

The second paper on three-spine stickleback, by D. I. Bolnick, considers the opposite conundrum: reproductive barriers are seemingly present but gene flow is not limited. Particularly, even though ecologically driven sympatric speciation does not always occur in sticklebacks, its theoretically necessary and sufficient conditions seem often to be present in nature. First, some populations experience strong competition for resources that causes extreme phenotypes to have higher fitness [28]. Second, assortative mating based on diet and morphology is present in some of these same lakes [29]. So how to solve this new conundrum? Using a simulation model, Bolnick demonstrates that the strengths of selection and assortative mating measured in lake populations in nature are too weak to cause sympatric speciation. Instead, lake stickleback appears to respond to disruptive selection through alternative means of reducing competition, such as increased genetic variance, sexual dimorphism, and phenotypic plasticity.

Another classic system for studying ecological speciation, or more generally adaptive radiation, is Anolis lizards of the Caribbean. In particular, many of the larger islands contain repeated radiations of similar "ecomorph" species in similar habitats [8]. Contrasting with this predictable and repeatable diversity on large islands, smaller islands contain only a few species. Y. Surget-Groba, H. Johansson, and R. S. Thorpe studied populations of Anolis roquet from Martinique. This species contains populations with divergent mitochondrial lineages, a consequence of previous allopatric episodes, and is distributed over a range of habitats. It can, therefore, be used to address the relative importance of past allopatry, present ecological differences, and their combination in determining progress toward ecological speciation. Using microsatellite markers, the authors find that geographic isolation alone does not result in significant population differentiation, habitat differences alone cause some differentiation, and geographic isolation plus habitat differences cause the strongest differentiation. The authors conclude that speciation is likely initiated in allopatry but is then completed following secondary contact only through the action of adaptation to different habitats.

Even though ecological differences are clearly important in the diversification of both plants and animals $[1,30]$, it remains uncertain as to whether the process is fundamentally the same or different between them. Part of the reason is that typical methods for studying ecological speciation differ between the two groups. In an effort to bridge this methodological divide, K. Ostevik, B. T. Moyers, G. L. Owens, and L. H. Rieseberg apply a common method of inference from animals to published studies on plants. In particular, ecological speciation is often inferred in animals based on evidence that independently derived populations show reproductive isolation if they come from different habitats but not if they come from similar habitats: that is, parallel speciation [31, 32]. Ostevik and coauthors review potential examples of ecological speciation in plants for evidence of parallel speciation. They find that very few plant systems provide such evidence, perhaps simply because not many studies have performed the necessary experiments. Alternatively, plants might differ fundamentally from animals in how ecological differences drive speciation, particularly due to the importance of behaviour in animals.

A current topic of interest in ecological speciation is whether strong selection acting on a single trait (strong selection) or relatively weak selection acting on a greater number of traits (multifarious selection) is more common and more likely to complete the speciation process [11]. Using another well-studied model of ecological speciation, butterflies of the genus Lycaeides, C. F. Scholl, C. C. Nice, J. A. Fordyce, Z. Gompert, and M. L. Forister compared host-plant associated larval performance of butterflies from several populations of $L$. idas, L. melissa, and a species that originated through 
hybridization between the two. By conducting a series of reciprocal rearing experiments, they found little to no evidence for local adaptation to the natal hosts. By putting these results into the context of the other previously studied ecological traits (e.g., host and mate preference, phenology, and egg adhesion $[33,34])$, the authors constructed a schematic representation of the diversification within this butterfly species complex. They conclude that no single trait acts as a complete reproductive barrier between the three taxa and that most traits reduce gene flow only asymmetrically. The authors suggest the need for further study of multiple traits and reproductive barriers in other taxa.

\section{Factors Affecting Progress toward Ecological Speciation}

4.1. The Role of Pollinators/Parasites. In many cases of ecological speciation, we think of the populations in question colonizing and adapting to divergent environments/resources, such as different plants or other food types. However, environments can also "colonize" the populations in question that might then speciate as a result. Colonization by different pollinators and subsequent adaptation to them, for example, is expected to be particularly important for angiosperms. A particularly spectacular example involves sexually deceptive Orchids, where flowers mimic the scent and the appearance of female insects and are then pollinated during attempted copulation by males. In a review and meta-analysis of two Orchid genera, S. Xu, P. M. Schlüter, and F. P. Schielst find floral scent to be a key trait in both divergent selection and reproductive isolation. Other traits, including flower colour, morphology and phenology, also appear to play an important role in ecological speciation within this group. The authors also conclude that although sympatric speciation is likely rare in nature, it is particularly plausible in these Orchids.

Parasites can be thought of as another instance of different environments "colonizing" a focal species and then causing divergent/disruptive selection and (perhaps) ecological speciation. As outlined in the contribution by A. Karvonen and O. Seehausen, differences in parasites could contribute to ecological speciation in three major ways. First, divergent parasite communities could cause selection against locally adapted hosts that move between those communities, as well as any hybrids. Second, adaptation to divergent parasite communities could cause assortative mating to evolve as a pleiotropic by-product, such as through divergence in MHC genotypes that are under selection by parasites and also influence mate choice (see also [35]). Third, sexual selection might lead females in a given population to prefer males that are better adapted to local parasites and can thus achieve better condition. The authors conclude that although suggestive evidence exists for all three possibilities, more work is needed before the importance of parasites in ecological speciation can be confirmed.

4.2. The Role of Habitat Choice. The importance of habitat (or host) isolation in ecological speciation is widely recognized. This habitat isolation is determined by habitat choice (preference or avoidance), competition, and habitat performance (fitness differences between habitats) [36]. S. E. Webster, J. Galindo, J. W. Grahame, and R. K. Butlin propose a conceptual framework to study and classify traits involved in habitat choice, based on three largely independent criteria: (1) whether habitat choice allows the establishment of a stable polymorphism maintained by selection without interfering with mating randomness or if it also promotes assortative mating; (2) whether it involves one-allele or twoallele mechanisms of inheritance; (3) whether traits are of single or multiple effect [37], the latter when habitat choice is simultaneously under direct selection and contributes to assortative mating. The combination of these three criteria underlies ten different scenarios, which the authors visit using previously published empirical data. They argue that the speed and likelihood of ecological speciation depends on the mechanism of habitat choice and at which stage of the process it operates, with scenarios of one-allele and/or multiple-effect traits being more favorable. While these scenarios have rarely been distinguished in empirical studies, Webster et al. reason that such distinctions will help in the design of future studies and enable more informative comparisons among systems. In practice, however, the identification of the mechanisms involved and discriminating among different scenarios may sometimes be difficult, as exemplified by the case of the intertidal gastropod Littorina saxatilis, a model system for ecological speciation.

Hybrids resulting from the crosses between individuals from populations with different habitat preferences will tend to show interest in both parental habitats. This will increase gene flow between parental species, inhibiting reproductive isolation. Inspired by host-specific phytophagous insects, J. L. Feder, S. P. Egan, and A. A. Forbes ask, what if individuals choose their habitat based on avoidance rather than preference? According to the authors, hybrids for alleles involved in avoidance of alternate parental habitats may experience a kind of behavioral breakdown and accept none of the parental habitats, generating a postzygotic barrier to gene flow. Feder and collaborators determine the reasons why habitat avoidance is underappreciated in the study of ecological speciation (theoretical and empirical), and try to improve this issue. They propose new theoretical models and do not find strong theoretical impediments for habitat avoidance to evolve and generate hybrid behavioral inviability even for nonallopatric scenarios. They also suggest a physiological mechanism to explain how habitat specialists evolve to prefer a new habitat and avoid the original one. Feder et al. also document empirical support for this theory. Accumulated data on Rhagoletis pomonella and preliminary results on Utetes lectoides strongly suggest that avoidance has evolved in these species, contributing to postzygotic reproductive isolation. A literature survey in phytophagous insects reveals at least ten examples consistent with habitat avoidance, and three cases of behavior inviability in hybrids consistent with this mechanism. The authors also present suggestions and cautionary notes for design and interpretation of results when it comes to experiments on habitat choice. 
Hybrid zones are particularly useful systems for determining whether differences in habitat preference or habitatassociated adaptation contribute to reproductive isolation. M. D. Carling and H. A. Thomassen investigate the effect of environmental variation on admixture in a hybrid zone between the Lazuli Bunting (Passerian amoena) and the Indigo Bunting (P. cyanea). They find that differences in environment explain interpopulation differences in the frequency and genetic composition of hybrids. This is not the first study to document an effect of environmental variation on the production or persistence of hybrids $[38,39]$ but Carling and Thomassen were also able to associate this pattern with specific environmental variables, particularly rainfall during the warmest months of the year. They discuss possible, complementary mechanistic explanations for these patterns, including habitat avoidance or preference in hybrids and habitat-dependent fitness. Their results indicate that inherent (i.e., non-geographic) barriers to gene flow between $P$. amoena and $P$. cyanea are environment dependent, which means these barriers could be ephemeral and vary in space and time.

S. P. Egan, G. R. Hood and J. R. Ott present one of the first direct tests of the role of habitat (host) isolation driven by host choice. Different populations of the gall wasp Belonocnema treatae feed on different oak species. Egan et al. first confirmed that $B$. treatae prefer their native host plant, with a stronger preference for females. They then demonstrated assortative mating among host populations, which was enhanced by the presence of the respective host plants. This enhancement was due to the fact that females usually mate on their host and that males also prefer their natal host plant. Therefore, host preference is directly responsible for reproductive isolation in $B$. treatae, by decreasing the pro-bability of encounter between individuals from different host populations. The mechanism revealed here likely applies to many host/phytophagous or host/parasite systems.

4.3. The Role of Phenotypic Plasticity. Phenotypic plasticity, the ability of a single genotype to express different phenotypes under different environmental conditions, has long been seen as an alternative to genetic divergence, and therefore as potential constraint on adaptive evolution [40, 41]. More recently, however, adaptive phenotypic plasticity has been rehabilitated as a factor potentially favoring divergent evolution by enabling colonizing new niches, where divergent selection can then act on standing genetic variation [42]. B. M. Fitzpatrick reviews the possible effects of phenotypic plasticity on the two components of ecological speciation: local adaptation and reproductive isolation. He finds that both adaptive and maladaptive plasticity can promote or constrain ecological speciation, depending on several factors, and concludes that many aspects of how phenotypic plasticity acts have been underappreciated.

Several other papers in the special issue also provide potential examples of the role of plasticity in ecological speciation. For instance, N. V. Vallin and A. Qvarnström studied habitat choice in two hybridizing species of flycatchers. When the two species occur in sympatry, pied flycatchers are displaced from their preferred habitat due to competition with the dominant collared flycatchers. Cross-fostering experiments showed that rearing environment matters to recruits' habitat choice more than does the environment of the genetic parents: pied flycatcher fledglings whose parents were displaced to pine habitats were more likely to return to nest in pine habitats. Thus, competition-mediated switches between habitats can cause a change of habitat choice through learning, which might then enhance reproductive isolation via ecological segregation. This role of plasticity and learning in habitat choice is also acknowledged in the contribution of Webster and collaborators.

\section{Unanswered Questions and Future Directions}

Although it is widely recognized that ecological speciation can occur without gene flow between diverging groups of individuals [43], the recognition of its importance has grown because of recent evidence for speciation with gene flow [44]. If gene flow commonly occurs during divergence, some mechanism, such as divergent selection must also occur frequently to counteract the homogenizing effect of gene flow. The manuscripts in this special issue, and a plethora of other recent publications [45-50], have made great strides in advancing our understanding of ecological speciation. These allow us to identify several key factors that affect progress toward ecological speciation, such as habitat choice (preference and avoidance), phenotypic plasticity, role of pollinators/parasites, complex biological interactions such as facilitation, as well as geographical context. However, for most cases, our understanding is still incomplete. For instance, the circumstances under which plasticity favors or inhibits adaptation, mate choice, and consequently ecological speciation are still largely unknown. Further insights will certainly arise from a multitude of empirical and theoretical studies, but certain areas of research are particularly likely to yield important results. For example, whereas we can rarely observe the time course of speciation in a single species, we can learn about factors affecting progress toward ecological speciation by studying and contrasting pairs of related populations at different points along the speciation continuum. Similarly, the study of parallel speciation may be highly informative. Such studies exist (e.g., [25, 26, 51, 52]), but we need many more systems where we can examine variation in progress toward ecological speciation. It is important that we also investigate instances where speciation fails, as these cases will advance our understanding of factors that constrain and enhance progress toward speciation. Furthermore, recent advances in DNA sequencing and statistical analysis offer an unprecedented opportunity to study the genetic basis and evolution of reproductive isolation during ecological speciation. The application of these new methods and models to ecologically well-studied systems have been and will be particularly informative $[53,54]$. Finally, more studies using experimental manipulations to study the effects of key parameters on ecological speciation are badly needed, especially if they can be combined with an understanding of natural populations. 


\section{Acknowledgments}

We would like to express our gratitude to all the authors and reviewers that contributed to the successful completion of this special issue. R. Faria research is financed by the Portuguese Science Foundation (FCT) through the program COMPETE (SFRH/BPD/26384/2006 and PTDC/BIA-EVF/ 113805/2009).

Marianne Elias

Rui Faria

Zachariah Gompert Andrew Hendry

\section{References}

[1] D. Schluter, The Ecology of Adaptive Radiation, Oxford University Press, Oxford, UK, 2000.

[2] P. Nosil, Ecological Speciation, Oxford University Press, Oxford, UK, 2012.

[3] H. D. Rundle and P. Nosil, "Ecological speciation," Ecology Letters, vol. 8, no. 3, pp. 336-352, 2005.

[4] K. Räsänen and A. P. Hendry, "Disentangling interactions between adaptive divergence and gene flow when ecology drives diversification," Ecology Letters, vol. 11, no. 6, pp. 624636, 2008.

[5] D. Lack, Darwin's Finches, Cambridge University Press, Cambridge, UK, 1947.

[6] G. G. Simpson, The Major Features of Evolution, Columbia University Press, New York, NY, USA, 1953.

[7] P. R. Grant and B. R. Grant, How and Why Species Multiply: The Radiation of Darwin's Finches, Princeton University Press, Princeton, NJ, USA, 2008.

[8] J. B. Losos, Lizards in an Evolutionary Tree: Ecology and Adaptive Radiation of Anoles, University of California Press, Berkeley, Calif, USA, 2009.

[9] R. J. Rundell and T. D. Price, "Adaptive radiation, nonadaptive radiation, ecological speciation and nonecological speciation," Trends in Ecology and Evolution, vol. 24, no. 7, pp. 394-399, 2009.

[10] A. P. Hendry, "Ecological speciation! Or the lack thereof?" Canadian Journal of Fisheries and Aquatic Sciences, vol. 66, no. 8, pp. 1383-1398, 2009.

[11] P. Nosil, L. J. Harmon, and O. Seehausen, "Ecological explanations for (incomplete) speciation," Trends in Ecology and Evolution, vol. 24, no. 3, pp. 145-156, 2009.

[12] J. B. Hughes, G. C. Daily, and P. R. Ehrlich, "Population diversity: its extent and extinction,” Science, vol. 278, no. 5338, pp. 689-692, 1997.

[13] G. E. Hutchinson, "Homage to Santa Rosalia or why are there so many kinds of animals?" The American Naturalist, vol. 93, pp. 145-159, 1959.

[14] J. Felsenstein, "Skepticism towards Santa Rosalia, or why are there so few kinds of animals?" Evolution, vol. 35, pp. 124-138, 1981.

[15] U. Dieckmann, J. A. J. Metz, M. Doebeli, and D. Tautz, "Introduction," in Adaptive Speciation, U. Dieckmann, J. A. J. Metz, M. Doebeli, and D. Tautz, Eds., pp. 1-17, Cambridge University Press, Cambridge, UK, 2004.

[16] R. B. Langerhans, M. E. Gifford, and E. O. Joseph, "Ecological speciation in Gambusia fishes," Evolution, vol. 61, no. 9, pp. 2056-2074, 2007.
[17] T. H. Vines and D. Schluter, "Strong assortative mating between allopatric sticklebacks as a by-product of adaptation to different environments," Proceedings of the Royal Society B, vol. 273, no. 1589, pp. 911-916, 2006.

[18] M. D. Bertness and R. Callaway, "Positive interactions in communities," Trends in Ecology and Evolution, vol. 9, no. 5, pp. 191-193, 1994.

[19] M. Kirkpatrick and N. H. Barton, "Evolution of a species' range," American Naturalist, vol. 150, no. 1, pp. 1-23, 1997.

[20] J. R. Bridle, J. Polechová, M. Kawata, and R. K. Butlin, "Why is adaptation prevented at ecological margins? New insights from individual-based simulations," Ecology Letters, vol. 13, no. 4, pp. 485-494, 2010.

[21] T. Dobzhansky, Genetics and the Origin of Species, Columbia University Press, New York, NY, USA, 1st edition, 1973.

[22] H. J. Muller, "Isolating mechanisms, evolution, and temperature," Biology Symposium, vol. 6, pp. 71-125, 1942.

[23] M. Turelli, N. H. Barton, and J. A. Coyne, "Theory and speciation," Trends in Ecology and Evolution, vol. 16, no. 7, pp. 330343, 2001.

[24] J. S. McKinnon and H. D. Rundle, "Speciation in nature: the threespine stickleback model systems," Trends in Ecology and Evolution, vol. 17, no. 10, pp. 480-488, 2002.

[25] A. P. Hendry, D. I. Bolnick, D. Berner, and C. L. Peichel, "Along the speciation continuum in sticklebacks," Journal of Fish Bio$\log y$, vol. 75, no. 8, pp. 2000-2036, 2009.

[26] H. D. Rundle, L. Nagel, J. W. Boughman, and D. Schluter, "Natural selection and parallel speciation in sympatric sticklebacks," Science, vol. 287, no. 5451, pp. 306-308, 2000.

[27] J. S. McKinnon, S. Mori, B. K. Blackman et al., "Evidence for ecology's role in speciation," Nature, vol. 429, no. 6989, pp. 294-298, 2004.

[28] D. I. Bolnick and L. L. On, "Predictable patterns of disruptive selection in stickleback in postglacial lakes," American Naturalist, vol. 172, no. 1, pp. 1-11, 2008.

[29] L. K. Snowberg and D. I. Bolnick, "Assortative mating by diet in a phenotypically unimodal but ecologically variable population of stickleback," American Naturalist, vol. 172, no. 5, pp. 733-739, 2008.

[30] J. M. Sobel, G. F. Chen, L. R. Watt, and D. W. Schemske, "The biology of speciation," Evolution, vol. 64, no. 2, pp. 295-315, 2010.

[31] D. J. Funk, "Isolating a role for natural selection in speciation: host adaptation and sexual isolation in Neochlamisus bebbianae leaf beetles," Evolution, vol. 52, no. 6, pp. 1744-1759, 1998.

[32] L. Nagel and D. Schluter, "Body size, natural selection, and speciation in sticklebacks," Evolution, vol. 52, no. 1, pp. 209218, 1998.

[33] J. A. Fordyce, C. C. Nice, M. L. Forister, and A. M. Shapiro, "The significance of wing pattern diversity in the Lycaenidae: mate discrimination by two recently diverged species," Journal of Evolutionary Biology, vol. 15, no. 5, pp. 871-879, 2002.

[34] J. A. Fordyce and C. C. Nice, "Variation in butterfly egg adhesion: adaptation to local host plant senescence characteristics?” Ecology Letters, vol. 6, no. 1, pp. 23-27, 2003.

[35] C. Eizaguirre and T. L. Lenz, "Major histocompatability complex polymorphism: dynamics and consequences of parasitemediated local adaptation in fishes," Journal of Fish Biology, vol. 77, no. 9, pp. 2023-2047, 2010.

[36] J. A. Coyne and H. A. Orr, Speciation, Sinauer, Sunderland, Mass, USA, 2004. 
[37] C. Smadja and R.K. Butlin, "A framework for comparing processes of speciation in the presence of gene flow," Molecular Ecology, vol. 20, pp. 5123-5140, 2011.

[38] D. M. Rand and R. G. Harrison, "Ecological genetics of a mosaic hybrid zone: mitochondrial, nuclear, and reproductive differentiation of crickets by soil type," Evolution, vol. 43, no. 2, pp. 432-449, 1989.

[39] T. H. Vines, S. C. Köhler, M. Thiel et al., "The maintenance of reproductive isolation in a mosaic hybrid zone between the fire-bellied toads Bombina bombina and B. Variegata," Evolution, vol. 57, no. 8, pp. 1876-1888, 2003.

[40] S. Wright, "Evolution in Mendelian populations," Genetics, vol. 16, pp. 97-159, 1931.

[41] D. A. Levin, "Plasticity, canalization and evolutionary stasis in plants," in Plant Population Ecology, A. J. Davy, M. J. Hutchings, and A. R. Watkinson, Eds., pp. 35-45, Blackwell Scientific Publications, Oxford, UK, 1988.

[42] D. W. Pfennig, M. A. Wund, E. C. Snell-Rood, T. Cruickshank, C. D. Schlichting, and A. P. Moczek, "Phenotypic plasticity's impacts on diversification and speciation," Trends in Ecology and Evolution, vol. 25, no. 8, pp. 459-467, 2010.

[43] D. Schluter, "Evidence for ecological speciation and its alternative," Science, vol. 323, no. 5915, pp. 737-741, 2009.

[44] C. Pinho and J. Hey, "Divergence with gene flow: models and data," Annual Review of Ecology, Evolution, and Systematics, vol. 41, pp. 215-230, 2010.

[45] P. Nosil, D. J. Funk, and D. Ortiz-Barrientos, "Divergent selection and heterogeneous genomic divergence," Molecular Ecology, vol. 18, no. 3, pp. 375-402, 2009.

[46] J. L. Feder and P. Nosil, "The efficacy of divergence hitchhiking in generating genomic islands during ecological speciation," Evolution, vol. 64, no. 6, pp. 1729-1747, 2010.

[47] J. L. Feder, G. Gejji, S. Yeaman, and P. Nosil, "Establishment of new mutations under divergence and genome hitchhiking,", Philosophical Transactions of the Royal Society B, vol. 367, pp. 461-474, 2012.

[48] J. L. Feder and P. Nosil, "Chromosomal inversions and species differences: when are genes affecting adaptive divergence and reproductive isolation expected to reside within inversions?" Evolution, vol. 63, no. 12, pp. 3061-3075, 2009.

[49] A. P. Michel, S. Sim, T. H. Q. Powell, M. S. Taylor, P. Nosil, and J. L. Feder, "Widespread genomic divergence during sympatric speciation," Proceedings of the National Academy of Sciences of the United States of America, vol. 107, no. 21, pp. 9724-9729, 2010.

[50] R. Faria, S. Neto, M. A. F. Noor, and A. Navarro, "Role of natural selection in chromosomal speciation," in Encyclopedia Life Sciences, Chichester, UK, 2011.

[51] D. Berner, A. C. Grandchamp, and A. P. Hendry, "Variable progress toward ecological speciation in parapatry: stickleback across eight lake-stream transitions," Evolution, vol. 63, no. 7, pp. 1740-1753, 2009.

[52] R. M. Merrill, Z. Gompert, L. M. Dembeck, M. R. Kronforst, W. O. Mcmillan, and C. D. Jiggins, "Mate preference across the speciation continuum in a clade of mimetic butterflies," Evolution, vol. 65, no. 5, pp. 1489-1500, 2011.

[53] P. A. Hohenlohe, S. Bassham, P. D. Etter, N. Stiffler, E. A. Johnson, and W. A. Cresko, "Population genomics of parallel adaptation in threespine stickleback using sequenced RAD tags," PLoS Genetics, vol. 6, no. 2, Article ID e1000862, 2010.

[54] P. Nosil and J. L. Feder, "Genomic divergence during speciation: causes and consequences," Philosophical Transactions of the Royal Society B, vol. 367, pp. 332-342, 2012. 

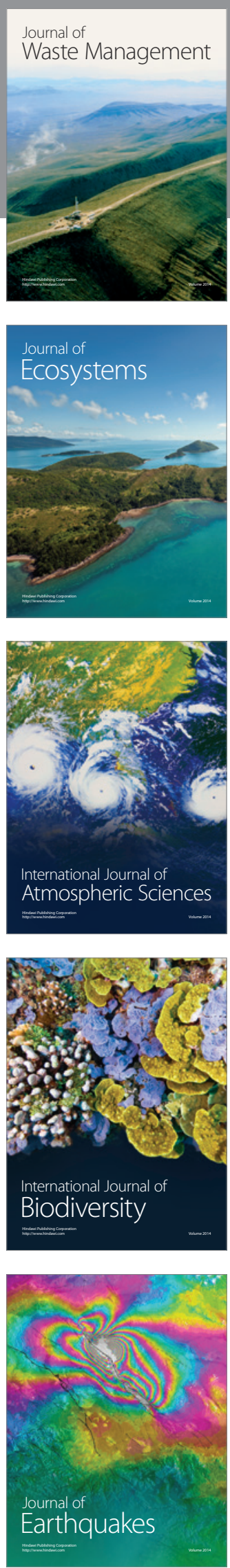
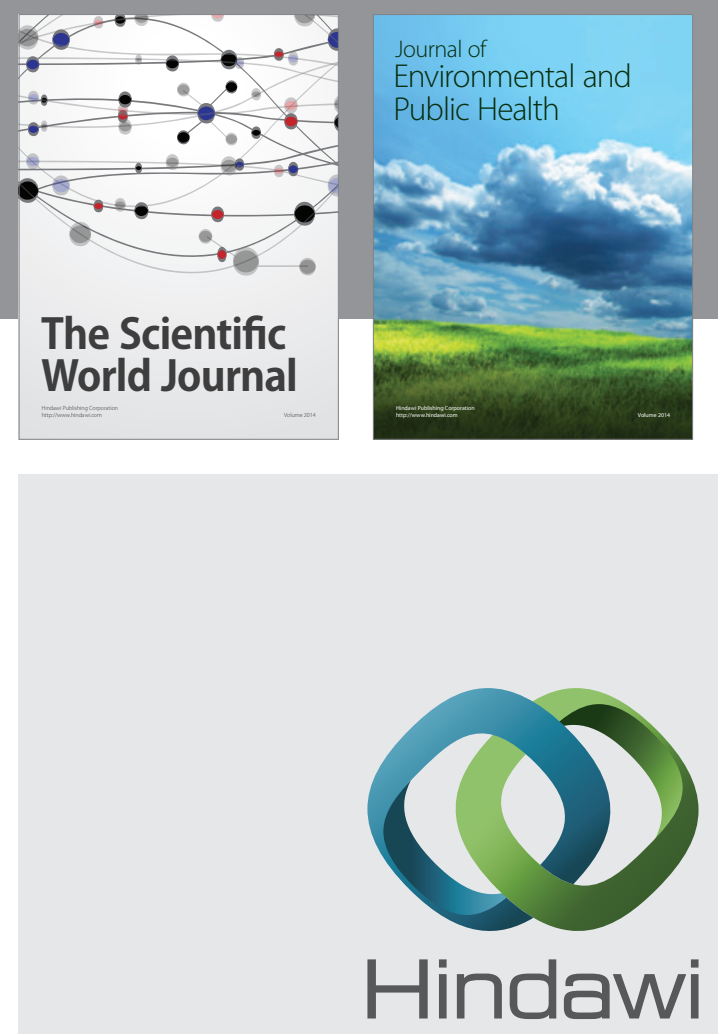

Submit your manuscripts at

http://www.hindawi.com
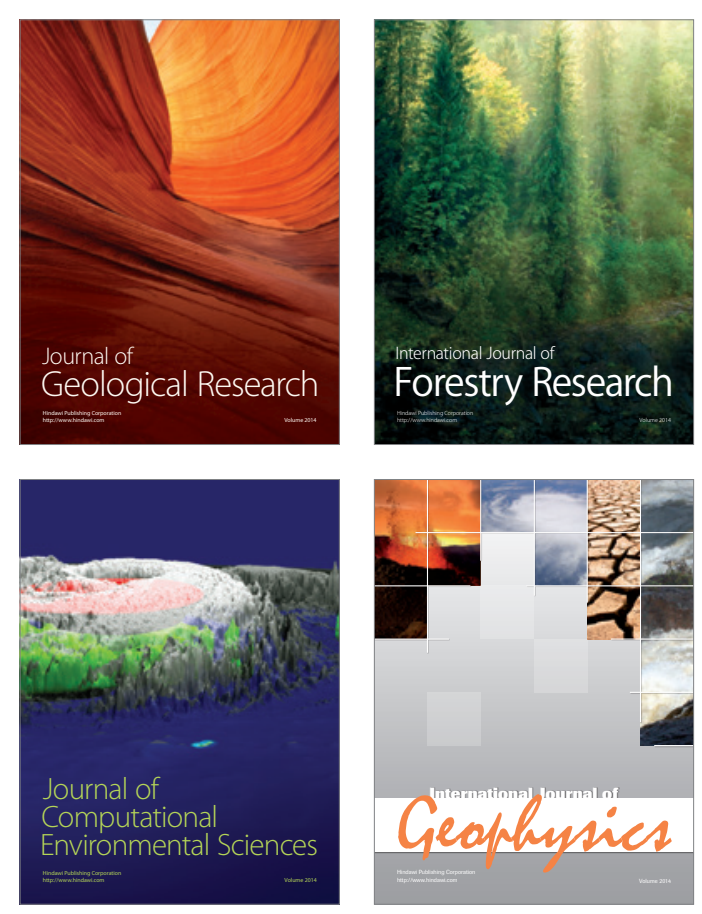
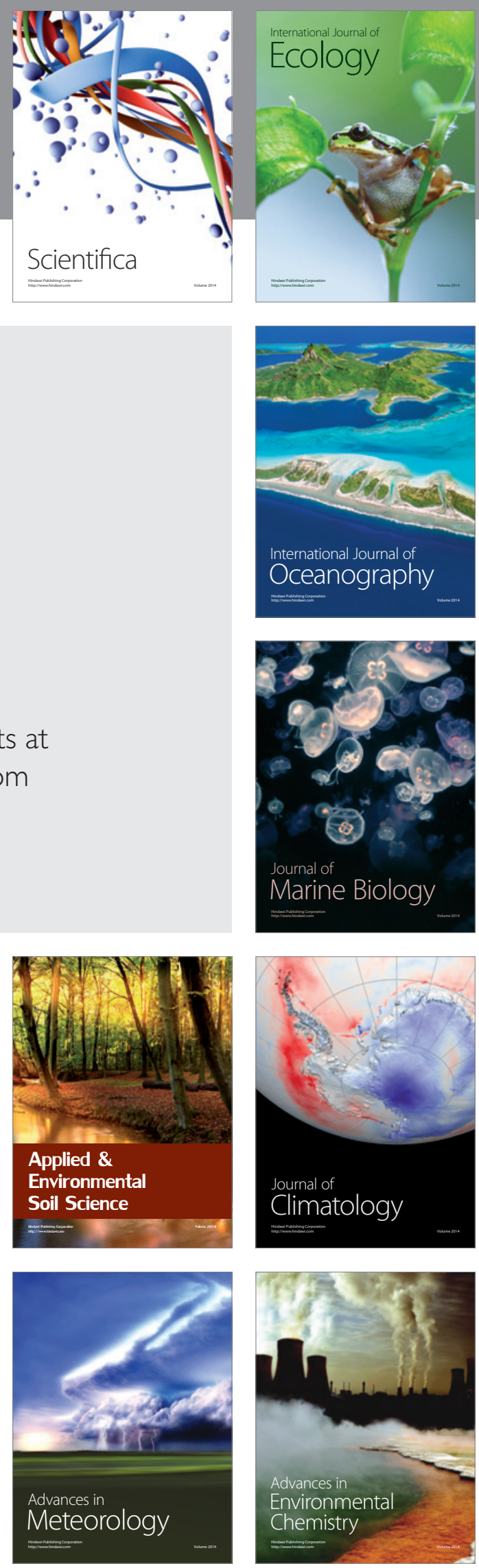